\title{
The Musical Kinetic Shape: A Variable Tension String Instrument
}

\author{
Ismet Handžić, Kyle B. Reed \\ University of South Florida, Department of Mechanical Engineering, Tampa, Florida
}

\begin{abstract}
In this article we present a novel variable tension string instrument which relies on a kinetic shape to actively alter the tension of a fixed length taut string. We derived a mathematical model that relates the two-dimensional kinetic shape equation to the string's physical and dynamic parameters. With this model we designed and constructed an automated instrument that is able to play frequencies within predicted and recognizable frequencies. This prototype instrument is also able to play programmed melodies.
\end{abstract}

Keywords: musical instrument, variable tension, kinetic shape, string vibration

\section{Introduction}

It is possible to vary the fundamental natural oscillation frequency of a taut and uniform string by either changing the string's length, linear density, or tension. Most string musical instruments produce different tones by either altering string length (fretting) or playing preset and different string gages and string tensions. Although tension can be used to adjust the frequency of a string, it is typically only used in this way for fine tuning the preset tension needed to generate a specific note frequency.

In this article, we present a novel string instrument concept that is able to continuously change the fundamental oscillation frequency of a plucked (or bowed) string by altering string tension in a controlled and predicted

Email addresses: ihandzic@mail.usf.edu (Ismet Handžić), kylereed@usf.edu (Kyle B. Reed)

URL: http://reedlab.eng.usf.edu/ () 

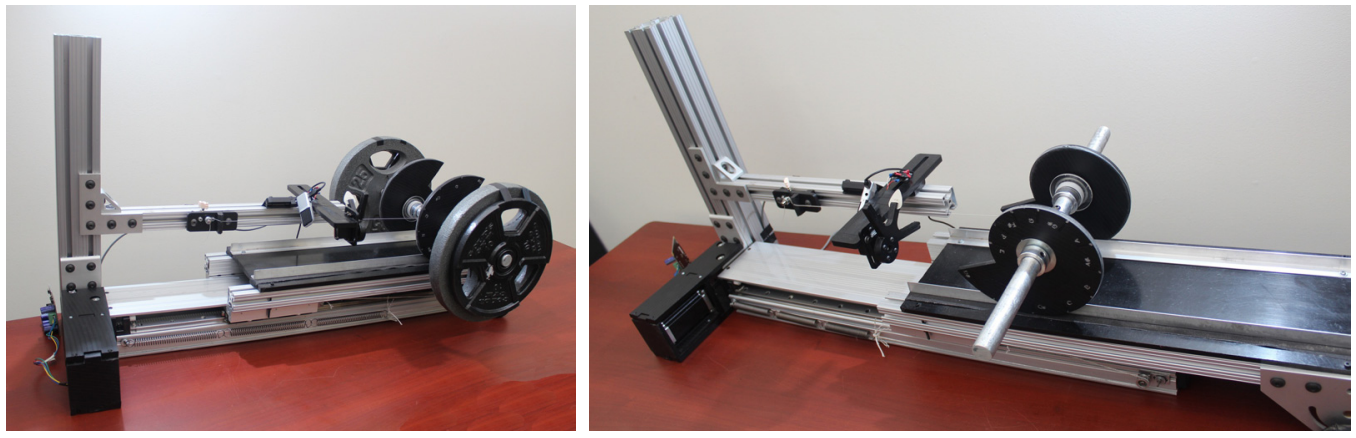

Figure 1: The musical kinetic shape variable tension string instrument prototype. In the image on the right, applied weights have been removed.

manner. We manipulate the tension in the string by attaching the string to the axle of a weight-loaded kinetic shape. A kinetic shape essentially acts as a mechanical force modifier redirecting an applied weight into a predicted ground reaction force with respect to the shape's orientation angle. As the weight-loaded kinetic shape changes orientation, the tension of the attached taut string produces a variation of tone on that plucked string. Our proposed string instrument setup can be seen in Figure 1.

A computer controlled stepper motor linearly moves a platform beneath the kinetic shape, re-orientating the shape at various angles. A constantlength guitar string is attached to the axle of the kinetic shape causing variable tension in the taut string as the shape is re-oriented. A thin guitar pick is attached to a computer controlled servo motor, plucking the string while a microphone records and analyzes the emitted sound.

In Section 3, we develop a mathematical formula which derives a kinetic shape that is dependent on an applied load and taut string parameters to yield predicted string oscillation frequencies as the string is plucked. The selection of these parameters which result in an appropriate kinetic shape is further discussed in Section 4, along with detailed instrument prototype design specifications. In Section 5 we compare predicted and measured string frequencies as the kinetic shape is repositioned. We also present the instrument's capability to play linear melodies. 


\section{Background}

\subsection{String Vibration}

In 1637, French mathematician and music theorist Marin Mersenne, who is often referred to as the "father of acoustics", used a sonometer [1] to derive formulas that predicted the lowest (fundamental) frequency of oscillation of a taut (stretched without sag) string [2]. These formulas, which relate string vibration frequency, mass per unit length, and tension, are refereed to as Mersenne's laws and are essential for the fabrication and operation of plucked, strummed, or bowed string instruments such as the guitar, chelo, piano, or harp. Marsenne's laws can be united as one formula that relates taut string vibration parameters and predicts a string's fundamental oscillation frequency (Equation 1).

$$
f=\left(\frac{1}{2 L}\right) \sqrt{\frac{T L}{\mu}}
$$

In Equation 1, $L$ is the length of the vibrating string, $T$ is the string tension, and $\mu$ is the mass per unit length of the string.

It is apparent that Marsenne's laws can be applied to produce different string vibration frequencies (pitch) by altering the string length, mass per unit length, or tension. That is, one can alter the vibration of an oscillating string by altering:

1. String Length (L): Holding all other factors constant, shorter strings will produce a higher vibration frequency, while longer strings will produce lower frequencies.

2. String Linear Density $(\mu)$ : A uniformly thicker string will move slower and so produce a lower frequency, while a thinner string will produce a higher frequency.

3. String Tension (T): Stretching a string with a higher force (tighter) will produce a higher frequency, while loosening the string will produce a lower frequency.

For example, all three strings depicted in Figure 2 will produce the same vibration frequency. In practice, however, the one parameter most used to vary string vibration frequencies is string length $(\mathrm{L})$.

Creating a variation of frequencies on one string by varying string length can be found on the guitar, violin, or chelo, where the player is able to 


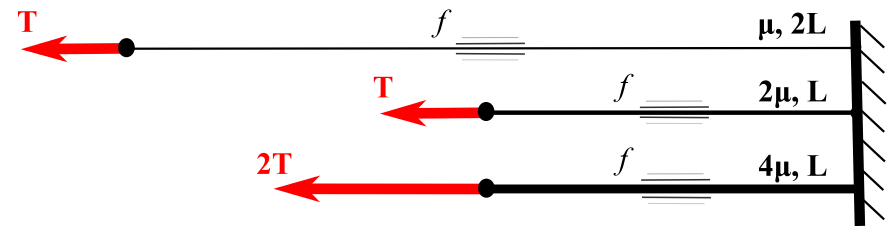

Figure 2: By Marsenne's laws, dissimilar combinations of taut string length, tension, and mass per unit length parameter can produce the same vibration frequency. Each of the strings shown will produce the same tone.

produce different tones on one string by changing the length of the string by fretting it against the instrument [3]. The traditional japanese kato (similar to zheng, yatga, or gayageum) [4] produces a variation of string vibration frequencies as the player changes string length by manually moving string supports (bridges).

Commonly, different notes can be produced by playing different gage (thickness) strings present on the same instrument, such as in the piano [5]. Usually preset string thicknesses are set on the instrument and do not actively change.

Although the string pitch may be altered by stretching, or "bending", the string in stringed instruments such as the guitar, which increases string tension, it is not the explicit way to play these instruments. String tension in stringed instruments is usually adjusted to calibrate, or fine tune, the instrument to a preset and unchanging tension.

The authors were only able to find one instrument that exclusively changes string vibration frequency by changing string tension. The bhapang [4]

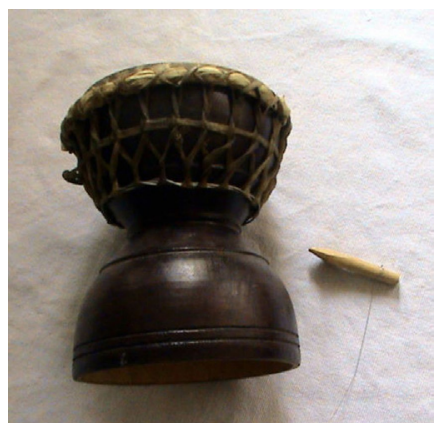

Figure 3: The bhapang is played by altering the string vibration frequency by tightening or loosening a string that is attached to a drum head. 
(Figure 3), is an Indian single stringed percussion instrument. The string, which is tightened or loosened by the player with a handle, passes through the drum head absorbing the drum's vibration as the drum is struck. The player can tighten or loosen the string to produce a continuous variation of sounds. Because of this continuous tension transition of the string, the pitch ramps up or down continuously.

It is also possible to automate and control a stringed musical instrument. This is not new concept and many mechatronic devices have been constructed to do so, which includes automating the piano [6] or violin [7]. A regularly performing thirteen piece robot musician band was developed that included seven guitars, two drum sets, two violins, and a cello, all controlled by electronic actuators [8]. In contrast, anthropomorphic robots have also been constructed and programmed to play instruments $[9,10]$.

A useful analytical application of the taut vibrating string concept is presented in geotechnical and civil engineering applications as vibrating strain gages $[11,12]$. These gages are placed into a soil, concrete, or onto structural members to measure deformations. As deformation occurs the tension in a taut wire is altered and, thus, the wire's oscillation frequency changes which is measured by a sensor.

Taut string vibration frequency as a function of tension can also be of great interest outside musical practices such as in mechanical analysis of medical equipment [13]. The Ilizarov apparatus is an external fixation device that is used to lengthen, reshape, or align bones. The Ilizarov apparatus frame is adjusted by tightening external tensioned wires. As guiding bone growth is a delicate process and misalignments can become permanent, the tensioned wires need to have exact specified tensions. These wire tensions can be found by wire vibration frequencies when plucked [13]. It was also found that after a few cycles of dynamic loading, pre-tension of these wires settle to a steady state tension [14].

\subsection{The Kinetic Shape}

It is possible to mechanically vary a force creating variable string tension through the use of a kinetic shape [15] attached to the string. To understand this concept, the kinetic shape is explained as follows.

A two-dimensional, smooth, rounded, and non-circular shape will roll when placed onto a horizontal surface. This rolling motion is due to the fact that the shape's applied weight does not horizontally line up with ground contact point, as seen in Figure 4a. As one applies more vertical downward 


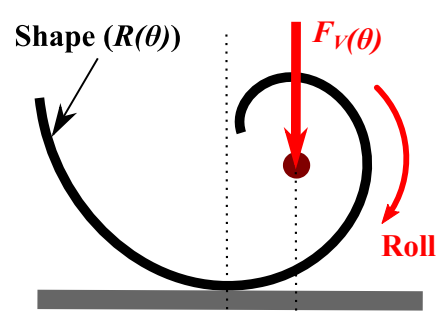

(a)

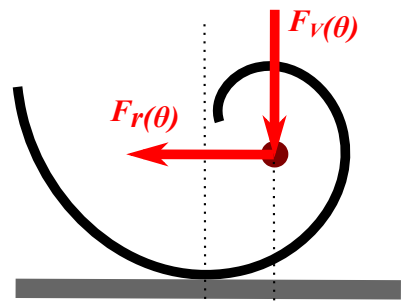

(b)

Figure 4: With a kinetic shape it is possible to derive a shape form which will yield a predicted horizontal reaction force given a known weight input.

force onto the shape rotation axis, the tendency to roll around the ground contact point becomes higher (rolling force increases). Assuming that the rolling motion is restricted by a horizontal force such that the shape does not roll and is in static equilibrium (Figure $4 \mathrm{~b}$ ). The horizontal force to keep the shape from rolling (static equilibrium) is dependent on the applied vertical force and on the shape form (curvature). A two-dimensional shape can be derived which when a known weight is applied to the shape, will exert a predicted horizontal force. This $2 \mathrm{D}$ kinetic shape equation as described in [15] is Equation 2.

$$
R(\theta)=\exp \left[\int \frac{F_{r}(\theta)}{F_{v}(\theta)} d \theta+\text { constant }\right]
$$

where $R(\theta)$ is the radius function that describes the shape in polar notation, $F_{v}(\theta)$ is the vertical force applied to the shape axle, and $F_{r}(\theta)$ is the horizontal force produced by the shape (tendency to roll). So given a constant vertical force (weight) applied to the shape rotation axis, it is possible to derive a shape, $R(\theta)$, to produce desired horizontal forces throughout the shape at different angles. Note that by Equation 2 the total dimensions of the kinetic shape are irrelevant and only the force ratio $\left(\frac{F_{r}(\theta)}{F_{v}(\theta)}\right)$ and shape curvature $\left(\frac{d R}{d \theta}\right)$ affects its behavior at any point around the shape. Applications of a kinetic shape range from rehabilitation devices [16] to the design of roll over shapes for prosthetics $[17,18]$.

\section{Theory}

As previously discussed, the vibration frequency of a taut string can be described with Equation 1, Marsenne's law, which relates string length, linear density, and tension. 


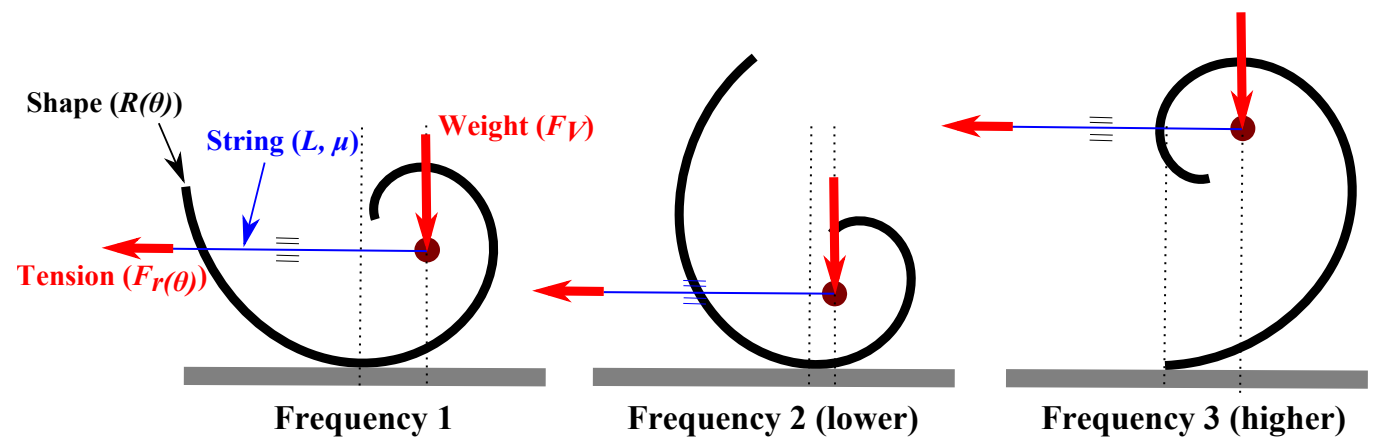

Figure 5: Due to the variable radius of curvature of the kinetic shape, repositioning the kinetic shape will cause different tensions in the taunt string and in turn cause the string to vibrate at different frequencies.

It is also common to specifically relate a keynote number to a note frequency. Keynote numbers are the conventionally designated numbers to discrete key sound frequencies. For example, the note $A 0$, which sounds at a frequency of $27.5 \mathrm{~Hz}$, has a keynote number of 1 , while the note $G \# 4$ has a frequency of $415.3 \mathrm{~Hz}$ and is referred as keynote number 48 . The relationship between keynote frequency $(f)$ and keynote number $(k)$ is given in Equation 3.

$$
f=2^{\frac{k-49}{12}} * 440
$$

The previously reviewed kinetic shape and string acoustic concepts can be combined to actively change the tension of a constant length string with constant linear density, and in turn produce different vibration frequencies of that string. This is possible if a kinetic shape axle is attached to a string, preventing it from rolling as a weight is applied to the kinetic shape's rotation axle. This setup can be seen in Figure 5.

The kinetic shape is actively rotated to specific positions around its perimeter to produce a predicted horizontal force (string tension), $F_{r}(\theta)$, which in turn produces different vibration frequencies in a plucked taut string (Figure 5). Note that the horizontal force, $F_{r}(\theta)$, applied by the kinetic shape onto the string is the string tension $(T)$ in Equation 1.

To correlate the shape form function, $R(\theta)$, string tension, $T$, and key note number, $k$, we first combine Equation 1 and Equation 3 to obtain a resulting horizontal force function (string tension function) exerted by the kinetic shape with respect to keynote number. 


$$
F_{r}(k)=\mu\left[880 L 2^{\frac{k-49}{12}}\right]^{2}
$$

where key note number, $k$, is a natural number. Equation 4 can also be presented as a continuous function between an initial and final keynote $n$ times around the kinetic shape.

$$
F_{r}(\theta)=\mu\left[880 L 2^{\frac{\theta}{\frac{2 \pi n}{2 \pi n}\left(k_{f}-k_{i}\right)+\left(k_{i}-49\right)}}\right]^{2}
$$

We then plug this tension function and a constant weight, $W$, as $F_{v}(\theta)$ into Equation 2 to obtain the form of the kinetic shape.

$$
R(\theta)=\exp \left[\int \frac{\mu\left[880 L 2^{\left.\frac{\theta}{\frac{2 \pi n}{\left(k_{f}-k_{i}\right)+\left(k_{i}-49\right)}}\right]^{2}}\right.}{W} d \theta+\text { constant }\right]
$$

After solving the indefinite integral, we yield

$$
R(\theta)=\exp \left[\frac{12 \pi n 880^{2} L^{2} \mu 2^{\frac{\theta\left(k_{f}-k_{i}\right)}{12 \pi n}+\frac{k_{i}-49}{6}}}{W\left[k_{f} \ln (2)-k_{i} \ln (2)\right]}+\text { constant }\right]
$$

Given an initial shape radius, $R(0)=R_{i}$, we are able to solve for the integration constant and obtain the final kinetic shape definition.

$$
R(\theta)=\exp \left[\frac{12 \pi n 880^{2} L^{2} \mu\left(2^{\frac{k_{i}-49}{6}}\right)\left(2^{\frac{\theta\left(k_{f}-k_{i}\right)}{12 \pi n}}-1\right)}{W\left[k_{f} \ln (2)-k_{i} \ln (2)\right]}+\ln \left(R_{i}\right)\right]
$$

Equation 8 defines a continuous radius of a kinetic shape from zero to $2 \pi n$, where given string parameters $(L, \mu)$, initial and final keynote numbers $\left(k_{i}, k_{f}\right)$, and an applied constant weight $(W)$ at the shape axle, will produce adequate string tension to provide the desired keynote string vibration frequencies. Since keynote angular positions are distributed around the derived kinetic shape. For a kinetic shape of $n$ revolutions (0 to $2 \pi n$ ), discrete keynotes angular positions $\left(\theta_{k}\right)$ are found using Equation 9 , where $k_{i}<k<$ $k_{f}$ and $k$ is a natural number. 


$$
\theta_{k}=\left(k-k_{i}\right) \frac{2 \pi n}{k_{f}-k_{i}}
$$

For example, on a kinetic shape that covers one revolution $(n=1)$ for initial keynote $k_{i}=10$ to final keynote $k_{f}=20$, keynote $k=15$ is found at angular position $\theta_{k}=\pi$.

\section{Instrument Design}

Our theory shows that by repositioning a kinetic shape attached to and tensioning a string, one can produce a variation of frequencies on that string. In this section we describe an automated prototype string instrument that is designed around this concept.

\subsection{Kinetic Shape Design and Fabrication}

The 2D kinetic shape equation (Equation 2) [15] indicates that the total dimensions of a kinetic shape are irrelevant, while only the curvature of the shape contributes to its behavior. However, preliminary tests concluded that in practice, a larger kinetic shape will produce more accurate results than an overall smaller one due to the fact that a smaller kinetic shape will be more affected by fabrication/surface imperfections and misalignment during use. Given all parameters, Equation 8 allows us to design a kinetic shape that produces a specified range of string vibration frequencies with adequate total shape dimensions.

For adequate accuracy, final kinetic shape, and (in turn) instrument dimensions, we selected the parameters presented in Table 1. The selection of these parameters was a process of trial and error using Equation 8 to determine the necessary range to play certain melodies. For example, in order to achieve the same keynote frequency range, choosing a lighter applied weight, longer string length, or heavier string would yield a larger radius change around the kinetic shape and vice versa. Note that the parameters chosen could be selected to cover different frequency ranges or to yield any overall size kinetic shape.

These chosen parameters are entered into Equation 8 to generate the shape shown in Figure 6. Note that it is possible to derive a kinetic shape for more than one revolution $(n>1)$, however, the curved rolling surface in such case would be more difficult to access with a flat and tangent surface. Also, unless specially fabricated, such a resulting kinetic shape could result 
Table 1: Parameters used to derive the instrument's kinetic shape.

\begin{tabular}{l||l} 
Shape Initial Radius $\left(R_{i}\right)$ & $2.5 \mathrm{in}(6.35 \mathrm{~cm})$ \\
\hline Revolutions $(n)$ & 1 \\
\hline Applied Weight $\left(F_{V}\right)$ & $82 \mathrm{lbf}(365 \mathrm{~N})$ \\
\hline String Length $(L)$ & 18 in $(45.7 \mathrm{~cm})$ \\
\hline String Linear Density $(\mu)$ & $0.0002159 \mathrm{lbm} /$ in $(0.00003856 \mathrm{~kg} / \mathrm{cm})$ \\
& Guitar String Type: D'Addario NW034 \\
\hline Initial Keynote $\left(k_{i}\right)$ & $19(D \# 2 / 77.8 \mathrm{~Hz})$ \\
\hline Final Keynote $\left(k_{f}\right)$ & $31(D \# 3 / 155.6 \mathrm{~Hz})$
\end{tabular}

in a less rigid structure. For ease of fabrication, robustness, and convenience we chose a kinetic shape that spans across one revolution $(n=1)$. Inserting parameters of Table 1 into Equation 5, we find that the string tension around the derived kinetic shape spans from $19.5 \mathrm{~N}\left(k_{i}=19\right)$ to $78.0 \mathrm{~N}\left(k_{f}=31\right)$.

The chosen two-dimensional kinetic shape was laser cut from a 0.375 " $(0.9525 \mathrm{~cm})$ thick sheet of tough Acetal Resin (Delrin $\left.{ }^{\circledR}\right)$ plastic. The laser cutter used to cut the shape was a 60 Watt Universal Laser System ${ }^{\circledR}$ VLS4.60. After cutting, the rolling surface of the kinetic shape was carefully sanded smooth to reduce any surface imperfections.

\subsection{Kinetic Shape Reorientation}

For our instrument, the derived kinetic shape has to be reorientated in a simple and accurate manner onto discretely defined points around the shape

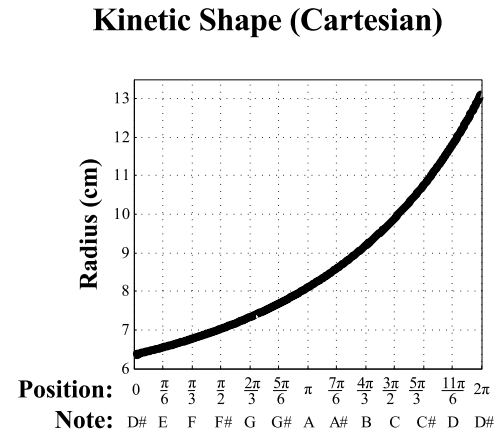

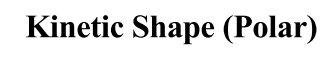

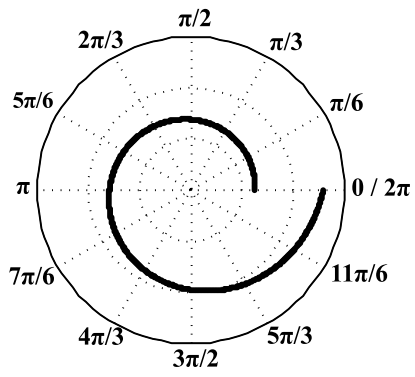

Kinetic Shape (Actual)

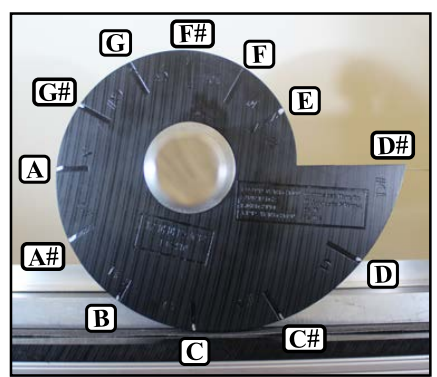

Figure 6: Derived kinetic shape to be used on prototype instrument. 


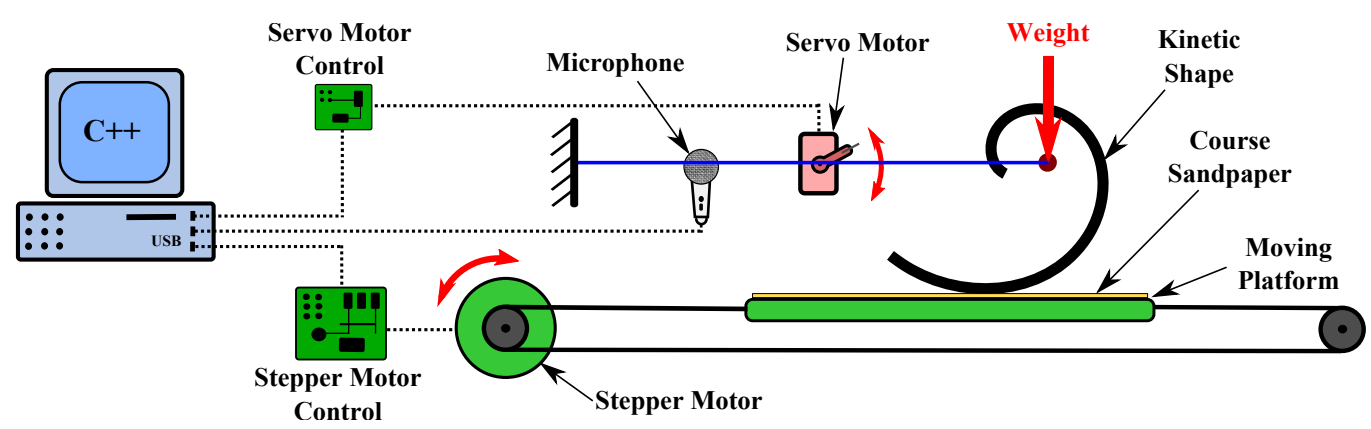

Figure 7: The complete schematic of the proposed string music instrument.

perimeter. Instead of repositioning the kinetic shape with respect to ground, a platform beneath the shape is moved, thus rolling the shape into position. To minimize error due to slippage between the kinetic shape rolling surface and the moving platform surface, course sandpaper was firmly screwed onto the moving surface. No soft material such as rubber could be utilized for the contact point between the shape and movable platform since the contact area needs to be as small as possible. To ensure accuracy the movable platform beneath the kinetic shape is actuated with a stepper motor. A schematic of this setup can be seen in Figure 7 . The stepper motor is firmly mated to a timing belt pulley which moves a tightened kevlar timing belt. The belt loops around an idler pulley to move a platform linearly on a smooth linear bearing.

The stepper motor was sized so that it can overcome the highest system torque, which is where the kinetic shape exerts the highest horizontal ground reaction force onto the movable platform $(\theta=2 \pi$ or $D \# 3)$. We chose a bipolar hybrid stepper motor with an $1.8^{\circ}$ resolution (MotionKing ${ }^{\circledR} 23 \mathrm{HS} 2430$ ). However, in the final design stages we added an extension spring in-line to the movable surface to provide additional force along with the stepper motor. The stepper motor was controlled by a Phidgets ${ }^{\circledR} 1067$ board that was interfaced with a $\mathrm{C}++$ program on a personal computer via USB.

Note that even without an electric motor it is easily possible to reorient the kinetic shape with the described setup by manually sliding the movable platform beneath the kinetic shape.

\subsection{Loading the Kinetic Shape}

For the shape to exert proper and predicted string tension, it cannot distribute any portion of the applied weight, $W$, onto its support structures. 

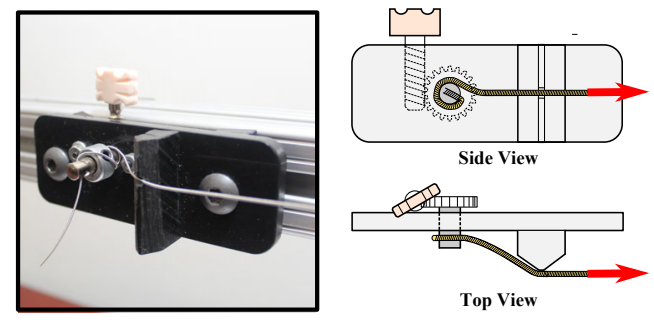

(a)
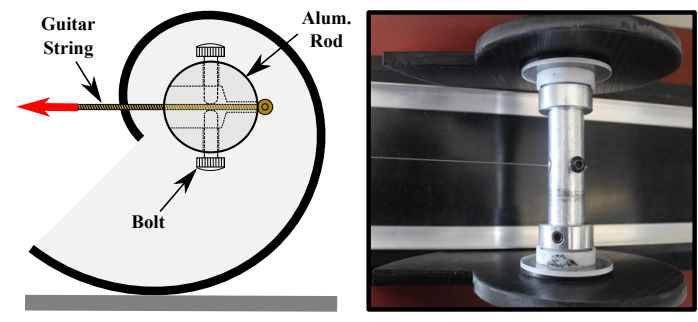

(b)

Figure 8: On one end the string is lengthened and shortened by a machine screw (worm gear mechanism), while the other end is fed through and fixed at the center of the aluminum rod.

Also, it must exert all ground reaction forces in the direction of the string vector. To alleviate this design constraint, two identical kinetic shapes were fabricated and positioned parallel to each other onto a $1.00 \mathrm{in}(2.54 \mathrm{~cm})$ aluminum rod with a fixed distance between them. Both kinetic shapes are held orthogonal with the rod, while being able to spin freely around their axle via smooth ball bearings. The actual setup of the two parallel kinetic shapes can be seen in Figure 1.

After the string is attached, weight is applied to the shape axles by placing discrete barbell weights on the outside of the kinetic shapes. For balance, the same amount of barbell weights are carefully placed on both sides. As the kinetic shapes are attached to the aluminum rod via ball bearings, the barbells do not rotate as the kinetic shape is rotated into different positions.

\subsection{Attaching a String}

Since we are using a steel guitar string, the two ends of the taut string are attached in a very similar fashion as a conventional electric guitar. The string's peg end is attached midway between the two parallel kinetic shapes. This end is pulled through a hole in the center of the aluminum rod, while it is held at rod center by two opposing set bolts as seen in Figure 8b. The other end is attached to a customized machine head (tuner, gear head) set at a fixed distance from the kinetic shape axle. The string passes over an elevated bridge, while it can be changed in length (lengthened or shortened) by the machine head for frequency calibration purposes (Figure 8a). Before usage, the kinetic shape was repositioned a number of times, dynamically loading and unloading the string, before the string assumed steady state length and tension. 


\subsection{String Plucking}

The string is plucked by an extra light/thin nylon guitar pick $(0.44 \mathrm{~mm})$, attached to a limited rotating servo motor that is held in position by an adjustable bracket. This servo motor (Phidgets ${ }^{\circledR}$ SM-S4505B) is controlled by a Phidgets ${ }^{\circledR} 1061$ servo controller board that is interfaced with a $\mathrm{C}++$ program on a personal computer via USB. The schematic of this setup is shown in Figure 7.

\subsection{Sound Recording and Analysis}

To verify and amplify the oscillation frequency of the string as the kinetic shape is reoriented, a microphone is placed in close proximity along the string to record emitted sound frequencies. The utilized microphone is the Samson ${ }^{\circledR}$ GoMic ${ }^{\circledR} 16 b i t / 44.1 k H z$ condenser USB microphone with a frequency range of $20 \mathrm{~Hz}$ to $18 \mathrm{kHz}$. The frequency range is well within our targeted frequency range from $77.8 \mathrm{~Hz}$ to $155.6 \mathrm{~Hz}$. After the string is plucked, the audio signal is recorded and a $\mathrm{C}++$ program computes the fast Fourier transform (FFT) while extracting the string's fundamental oscillation frequencies in real time.

\subsection{Playing a Melody}

The $\mathrm{C}++$ program is also able to be programed to reorient the kinetic shape to manually or automatically play keynote frequencies in a linear succession with a specified rhythm by taking into account the time it takes to reposition the shape. In other words, the program allows the instrument prototype to play simple melodies. This prototype is only able to play linear note sequences, because only one string is plucked at one time. The instrument is also able to play vibratos by simply rocking the kinetic shape back and forth, increasing and decreasing the tension in the taut string.

\section{Results}

\subsection{Shape Position Versus Frequency}

Once our musical kinetic shape string prototype instrument was assembled and dynamically loaded, it was calibrated to known frequencies around the kinetic shape by slightly lengthening or shortening the guitar string. After calibration the shape was automatically oriented from $\theta=\pi / 6(E 2)$ to $\theta=11 \pi / 6(D 3)$ at 20 even intervals. $\theta=0(D \# 2)$ and $\theta=2 \pi(D \# 3)$ were not tested due to inaccessibility (see Figure 6). At each step the string was 


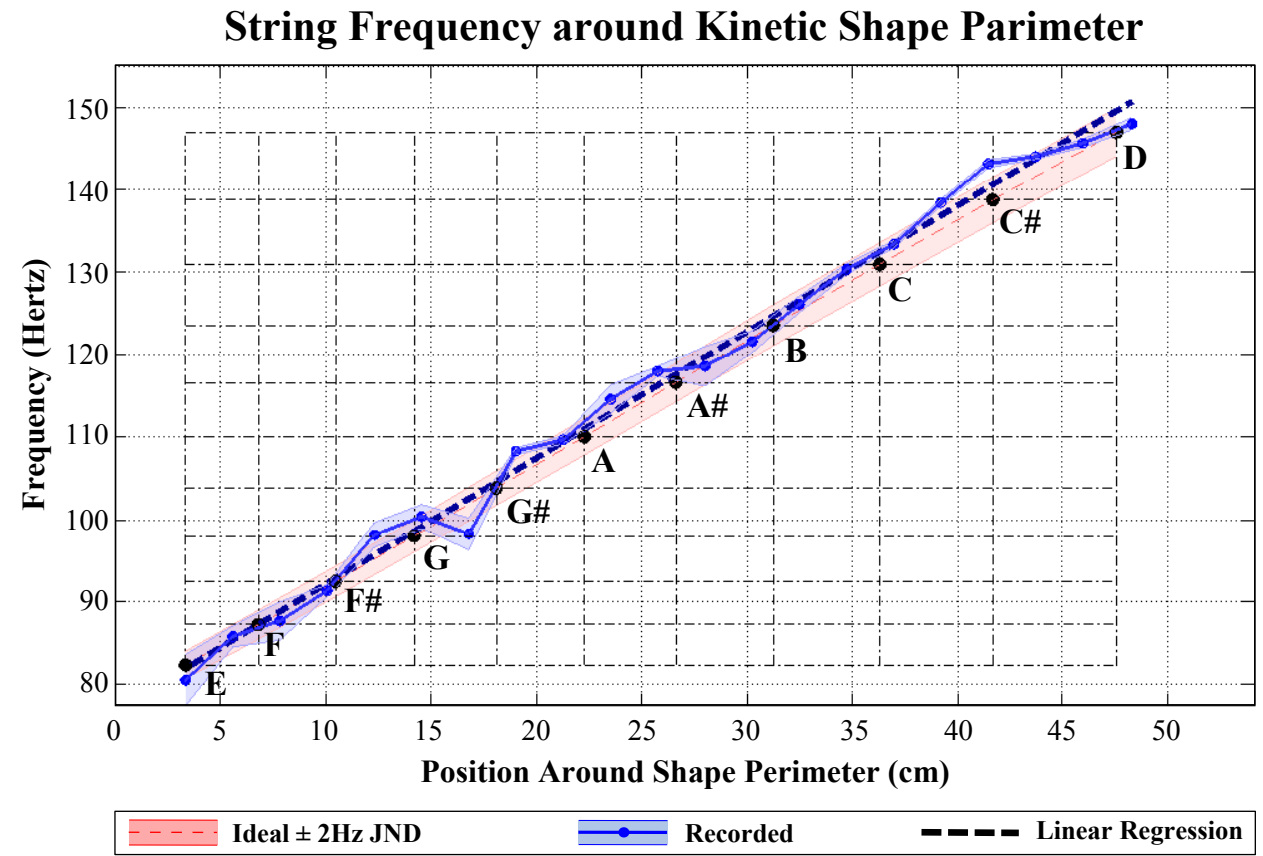

Figure 9: A guitar string was plucked at twenty different positions around the kinetic shape while the string vibration frequency was recorded. The shaded band is the standard deviation of all readings at that particular position.

plucked ten times with three seconds between plucks, while the dominant string oscillation frequency was recorded at each pluck. After ten plucks the average and standard deviation for one shape orientation was computed and the stepper motor moved the shape into the next orientation. Figure 9 shows the recorded frequencies as a function of positions around the kinetic shape's perimeter.

As a standard we compare our recorded frequencies to expected frequencies with an offset of the human ear's just noticeable difference (JND) for pitch. JND (or differential threshold) of the ear is the smallest detectable difference in pitch that the human ear can detect. Although this JND varies depending upon frequency, sound level, and sound duration, the JND for frequencies below $500 \mathrm{Hertz}$ is generally found to be $2 \mathrm{Hertz}$ [19].

Despite that the recorded frequencies around the kinetic shape vary slightly from ideal, they are mostly within the JND range. That is, the average human ear could not detect the difference between ideal frequency and the frequency produced by our instrument. The jumps and variations in recorded 

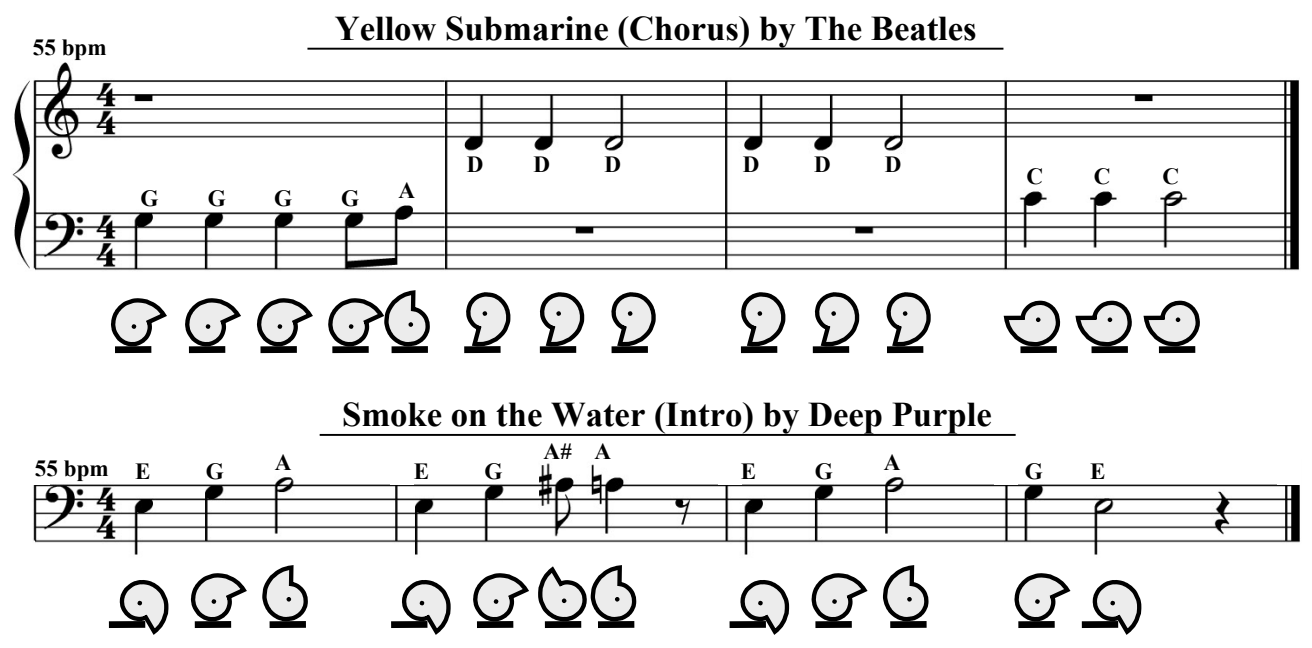

Figure 10: The musical kinetic shape instrument is programmed to automatically reorient the kinetic shape at even time intervals (beats) to produce two separate melodies.

frequencies can be accounted by imperfections in surface contact between the kinetic shapes and the movable platform and slight misalignment between the two parallel kinetic shapes.

\subsection{Melody Play}

Since the constructed prototype instrument is able to play melodies that include available notes $E, F, F \#, G, G \#, A, A \#, B, C, C \#$, and $D$, we chose two melodies that include these notes to be played by the musical kinetic shape prototype instrument. The chosen melodies are presented in Figure 10 and are shown in the supplemental video that accompanies this manuscript. The prototype was unable to play the melodies at full original tempo due to the fact that faster movement of the kinetic shape required more power than the stepper motor could provide. Larger accelerations and decelerations also accounted for slight slippage at the contact surface between the kinetic shape and movable platform. Melodies were played at $55 \mathrm{bpm}$, which is roughly half of the songs' original playing tempo.

However, although the melodies were played at half tempo, the notes were precisely timed and played at the correct frequency throughout the two melodies. It is interesting to note that after a longer period of usage, the instrument had to be calibrated due to contact surface slippage, but adding a feedback controller would allow the instrument to be continuously calibrated in real time. 


\section{Conclusion and Future Work}

We have successfully derived the theory and constructed a prototype for a variable tension musical kinetic shape string instrument. Our prototype produced frequencies which were within ideal and recognizable vibration frequencies throughout the instrument's range, however slight variation persisted due to possible misalignments and slippage of the kinetic shape surface. This type of slippage could possibly be alleviated by coating the movable platform with higher friction material or even gearing the kinetic shape.

The instrument also successfully performed two simple melodies at $55 \mathrm{bpm}$ at precise timing and frequencies. More complicated and faster melodies and even chords could be produced by having two or more strings with corresponding kinetic shapes parallel to each other reoriented independently and played simultaneously. For example, as one string-shape is being played other string-shapes rotate into position for upcoming notes. Although our pilot design embodies a string that is being plucked, it is also possible to have the same instrument by bowing the string in variable tension.

While our prototype only utilizes one revolution around a kinetic shape, it is possible to design a very similar instrument with a greater range by creating a kinetic shape with more than one revolution, or even a 3D kinetic shape [15] that is attached to two string with two independent tensions.

Although this prototype is used to generate music, the same concept can be applied to manufacture strain gages that have adjustable sensitivity. This could be done by placing the kinetic shape into a soil, concrete, or other medium such that the deformed medium applies a force onto the shape which tightens or loosens a vibrating wire.

\section{References}

[1] J. Hunt, A vertical sonometer, American Journal of Physics 58 (1990) 93.

[2] M. Mersenne, Harmonice Universelle, 1636.

[3] T. D. Rossing (Ed.), The Science of String Instruments, Springer, New York, 2010.

[4] K. Hunt, S. Broughton, "Everything Is Left Behind" in the Rough Guide to World Music, Rough Guides, London, 2000. 
[5] J. Harold A. Conklin, Design and tone in the mechanoacoustic piano. part iii. piano strings and scale design, Acoustical Society of America 100 (1996) 1286-1298.

[6] E. Hayashi, Automated piano: Techniques for accurate expression of piano playing, in: J. Solis, K. Ng (Eds.), Musical Robots and Interactive Multimodal Systems, Springer, 2011, pp. 143-163.

[7] K. Shibuya, Violin playing robot and kansei, in: J. Solis, K. Ng (Eds.), Musical Robots and Interactive Multimodal Systems, Springer, 2011, pp. 179-193.

[8] T. M. Sobh, B. Wang, K. W. Coble, Experimental robot musicians, Journal of Intelligent and Robotic Systems 38 (2003) 197-212.

[9] I. Kato, Devlopment of WABOT 1, Biomechanism 2 (1973) 173-214.

[10] I. Kato, WABOT-2: Autonomous robot with dexterous finger arm, Proc. IEEE Robotics Automation 5 (1987).

[11] C. O. Hunt, K. William, Vibrating strain gauges (patent US3052116A), 1962.

[12] I. Hawkes, Vibratory wire strain gage (patent US4277973A), 1981.

[13] V. L. Russa, B. Skallerud, J. Klaksvik, O. A. Foss, Wire tension versus wire frequency: An experimental ilizarov frame study, Journal of Biomechanics 43 (2010) 2327-2331.

[14] A. Renard, B. Schutte, N. Verdonschot, A. V. Kampen, The ilizarov external fixator: What remains of the wire pretension after dynamic loading?, Clinical Biomechanics 20 (2005) 1126-1130.

[15] I. Handžić, K. B. Reed, Kinetic shapes: Analysis, verification, and application, ASME Journal of Mechanical Design 137 (2014) (under review).

[16] I. Handžić, E. Barno, E. V. Vasudevan, K. B. Reed, Design and pilot study of a gait enhancing mobile shoe, J. of Behavioral Robotics 2 (2011) 193-201. 
[17] A. Hansen, D. Childress, E. Knox, Prosthetic foot roll-over shapes with implications for alignment of trans-tibial prostheses, Prosthetics and Orthotics International 24 (2000) 205-215.

[18] A. Hansen, C. Wang, Effective rocker shapes used by able-bodied persons for walking and fore-aft swaying: Implications for design of ankle-foot prostheses, Gait and Posture 32 (2010) 181-184.

[19] B. Kollmeier, T. Brand, B. Meyer, Perception of Speech and Sound, Springer handbook of speech processing, Springer, 2008. 